\title{
Alfred Graefe und die Antisepsis in der Augenheilkunde
}

von Manfred Tost

\section{Zusammenfassung}

Die Einführung des Listerschen Verfahrens der Antisepsis in die Augenchirurgie ist ein Verdienst Alfred Graefes (1830-1899). Nach kurzem biographischem Überblick und Hinweisen auf die Originalarbeiten zum Thema werden die zum Teil kontroversen Diskussionen besprochen. In das wissenschaftiche Umfeld wird das gute Verhältnis Alfred Graefes zu Richard v. Volkmann (1830-1889), damals Ordinarius für Chirurgie in Halle, einbezogen, auf den entscheidende Bemühungen um die Einführung der Antisepsis in der Allgemeinchirurgie zurückgehen.

Die entscheidenden Entwicklungsprozesse der Ophthalmologie und der Bakteriologie sind bemerkenswerterweise zeitlich der beginnenden zweiten Hälfte des 19. Jahrhunderts zuzuordnen. Während sich in der Augenheilkunde mit der Erfindung des Augenspiegels durch Helmholtz (1821-1894) Grundlegendes in der Diagnostik des Augenhintergrundes erschloss, so ist in der Bakteriologie die Entdeckung der Mikroorganismen durch Louis Pasteur (1822-1895) als Voraussetzung für die Inauguration antiseptischer Verfahren anzusehen. Es ist Joseph Lister (1827-1912) zu verdanken, die antibakterielle Wirkung der Karbolsäure im Sinne einer chirurgischen Antisepsis 1867 empfohlen zu haben.

Die unzweifelhaften Erfolge der Listerschen Methode gaben nunmehr den Anlass, diese auch in den Tochterdisziplinen der Chirurgie, namentlich der Augenheilkunde, zu erproben. Die wachsende Anerkennung der Ophthalmologie in jener Zeit zeigt sich u.a. darin, dass die Gründung der meisten deutschen Ordinariate dieses Faches in die frühen siebziger Jahre des 19. Jahrhunderts fiel. Die Zielsetzung war in der Senkung der Komplikations- bzw. Verlustrate bei ophthalmo-chirurgischen Eingriffen zu suchen. Eine Enophthalmitis septica - auch Suppuration genannt - war in der damaligen Zeit einer Erblindung gleichzusetzen. Noch 1888 beklagte Bjerrum, dass unter 253 Patienten mit unkomplizierter seniler Katarakt fünf an Entzündungen verstarben und drei weiteren das Sehvermögen verlorenging. 
Dem an sich logischen Entschluss zur Einführung der Antisepsis in der Ophthalmologie standen zwei wichtige Positionen gegenüber: Zum einen schienen operative Eingriffe am Auge im allgemeinen nicht extrem mit mikrobiellen Zwischenfällen belastet zu sein. Zum anderen war die Anwendung ätzender Substanzen von einer mehr oder minder schweren Schädigung von Lidern, Bindehaut und Hornhaut gefolgt, die nun ihrerseits bei konsekutiver Narbenbildung eine unter Umständen erhebliche Funktionseinbusse nach sich zog.

Eine ausreichende antiseptische Wirkung bei vertretbarer objektiver und subjektiver Belastung des Patienten erzielt zu haben, ist das besondere Verdienst Alfred Graefes, der 1873 als Ordinarius für Ophthalmologie auf den neu gegründeten Lehrstuhl an der Medizinischen Fakultät der Vereinigten Friedrichs-Universität zu Halle berufen worden war.

Der Werdegang Alfred Graefes, geboren am 23. November 1830 auf Schloss Martinskirchen bei Mühlberg/Elbe, sei nur kurz skizziert: Schulbildung an den Franckeschen Stiftungen in Halle/Saale, Medizinstudium in Halle, Heidelberg, Würzburg, Leipzig und Prag. 1854 Dissertation mit dem Thema «De canaliculorum lacrymalium natura», Hospitation in Paris bei Julius Sichel (1802-1868) und Louis-Auguste Démarres (1819-1882), weitere Ausbildung bei seinem Vetter Albrecht v. Graefe (1828-1870) in Berlin von 1855 bis 1858. Habilitation in Halle 1858 mit der Arbeit «De signis ophthalmoscopicis quorundam amblyopiae generum quae ad retinae morbos referenda sunt». Inhaber des Ordinariates von 1873 bis 1892 . Gestorben am 12. April 1899 in Weimar. Hauptarbeitsgebiet: Motilitätsstörungen des Auges. Zusammen mit Theodor Saemisch Herausgeber des ersten deutschsprachigen Handbuches der gesamten Augenheilkunde 1874-1880 (Waldemeyer 1981).

Im genannten Handbuch Graefe-Saemisch findet sich entsprechend der damaligen wissenschaftlichen Entwicklung noch kein Hinweis auf die Bedeutung des Listerschen Verfahrens für die Augenheilkunde. Doch schon 1878 veröffentlicht Alfred Graefe die Ergebnisse der antiseptischen Wundbehandlung bei Kataraktextraktionen in Albrecht v. Graefes Archiv für Ophthalmologie. Er hält die «pure Übertragung des Listerschen Verfahrens auf unser Feld» für unstatthaft, trägt bei Augenoperationen $2 \%$ ige Karbolsäurelösung auf die Lidflächen und das Auge selbst mit dem Schwämmchen auf, verabfolgt nach der Operation 4\%ige Borsäurelösung und einen Verband mit Listerschem Borsäure-Lint. Bei 144 Kataraktoperationen kann Alfred Graefe (1878) die Verlustquote von 5-6\% (im Mittel) auf 236/57 \% senken. 
Die Arbeiten Graefes und anderer Mitstreiter werden, wie nicht anders zu erwarten, auch kritisch beurteilt. Eine Reihe von Autoren hält nach klinischen Beobachtungen, z. T. auch nach tierexperimentellen Untersuchungen, die Karbolsäure-Anwendung am Auge für nachteilig oder zumindest strittig. Angeführt werden Hornhauttrübungen, Reizungen von Binde- und Lidhaut, erhebliche subjektive Beschwerden, das Verderben der Instrumente und anderes (Horner 1874, Strasser 1880). Graefe verschliesst sich nicht, selbst nachteilige Befunde mitzuteilen, wie z.B. «erysipelartige Schwellungszustände» der Augenlider (1878).

Um die Nebenwirkungen von Karbolsäure zu reduzieren, suchte man nach anderen Antiseptika und empfahl z. B. Borsäure, Natrium benzoicum, Sublimat, Borglyzerin und Salizylsäure. Alfred Graefe hat sich an diesen Bemühungen mit weiteren umfassenden Untersuchungen beteiligt. So berichtet er 1884 über vier differente antiseptische Methoden, deren Einsatz und Auswertung auf der beachtlichen Anzahl von 1419 Starextraktionen beruhte. Neben Karbolsäure kamen in getrennten Untersuchungsreihen Borsäure bzw. Sublimat zur Verwendung.

Die Prozentangaben für den Totalverlust des Sehvermögens durch «Suppuration» sind in der folgenden Tabelle 1 zusammengestellt.

Tab. 1: Totalverlust des Sehvermögens durch «Suppuration» (nach Angaben von A. Graefe 1884)

$\begin{array}{ll}\text { Zahl der } & \text { Verluste } \\ \text { Operationen } & \text { in Prozent }\end{array}$

\section{Beobachtungsreihe 1}

Abwaschen der Lider und der Kon-

junktiva mit Karbolsäure $2 \%$ ig

$5^{255 / 499}$

\section{Beobachtungsreihe 2}

Karbolsäurespray $2 \%$ ig

$6^{132 / 413}$

\section{Beobachtungsreihe 3}

Abwaschen der Lider und der Konjunktiva mit Borsäure 4\%ig

\section{Beobachtungsreihe 4}

Spülung mit Sublimatlösung

$1: 5000$ 
Die beim Vergleich der Arbeit von 1878 mit der von 1884 erscheinenden Differenzen dürften durch die ungleich grossen Patientengruppen, aber auch durch zufällige andere Ursachen zu erklären sein. In seiner 1889 erschienenen Arbeit findet man sogar die Angabe, dass «448 hintereinander ohne einen Fall von Suppuration operiert wurden» (Sublimat-Desinfektion).

Ungeachtet des tatsächlichen Fortschrittes in der operativen Behandlung der Katarakte durch die angeführten antiseptischen Methoden war sich Graefe der kritischen Deutung seiner Ergebnisse von Anbeginn bewusst:

«Nur kurz will ich bemerken, dass, falls man auch hier an eine jener guten Launen des Schicksals denken möchte, wie sie uns periodisch ja zu lächeln scheinen, unter den meinen Mittheilungen zu Grunde gelegten Fällen auch eine grosse Reihe solcher sich befand, welche einer derartigen Gunst des Zufalls in hohem Grade bedurft hätten, da sie nach den geltenden Anschauungen zur Suppuration vorzugsweise disponirt erschienen.» (Alfred Graefe 1878)

Manche Ablehnung des Listerschen Verfahrens entbehrt nicht des sarkastischen Untertones, wenn man z. B. im Referat einer Arbeit Bribosias (1880) liest :

«Gegen den Rath von Snellen hat B. den Spray mit Karbolsäure (von Lee) sogar «Stunden lang) vor der Operation angewendet, - hoffentlich in Abwesenheit von Arzt und Patienten». B. wird gefragt, ob der Spray nicht etwas Mystisches wäre.

Befürwortung und kontroverse Diskussion antiseptischer Massnahmen haben als einen unbedingt herauszustellenden Vorteil eine Vielfalt von Empfehlungen zum Operationsregime im weitesten Sinne im Gefolge gehabt. Sauberkeit von seiten des Patienten und des betreuenden Personals, Kontrolle der Instrumente, peinliche Sterilisation, entsprechend vorgerichteter Operationssaal, Einwegmaterialien (!), Trennung von primär septischen und aseptischen Operationen, Einzelzimmer für die Frischoperierten sind bereits in den achtziger Jahren des vorigen Jahrhunderts formuliert und gefordert worden (Abadie 1885, Birnbacher 1885, Kümmel 1885, Panas 1885, Rosenzweig 1885). Diese Prämissen haben im Grunde noch heute Gültigkeit in der Ophthalmologie. Manches ist hinsichtlich des Vorgehens noch immer nicht abschliessend entschieden. Welches Fachgebiet - die allgemeine Chirurgie oder die Ophthalmologie - den primären Anspruch auf die Einführung allgemeiner Hygieneempfehlungen für operative Eingriffe erheben darf, ist nicht zu klären. Noch bei Steffen lesen wir 1889:

«Wie Jacobsohn (1884) zu der kühnen Behauptung kommt, die Ophthalmologen hätten erst von den Chirurgen die Reinlichkeit als Bedingnis guter Wundheilung lernen müssen, ist 
mir unerklärlich. Das Umgekehrte würde wohl der Wahrheit näher kommen. Hätten die Chirurgen von jeher mit gleicher sorgfältiger Reinlichkeit, wie die Ophthalmologen stets gewohnt waren, operiert, so wäre bei ihnen das Bedürfnis und der Erfolg der Antisepsis wohl kein so überraschender gewesen.»

Mit Sicherheit aber sind Sauberkeit und Vorsicht in der Ophthalmologie nicht durch «Antiseptik» - und man möchte heute hinzufügen, auch nicht durch «prophylaktische Antibiotikagaben» - zu ersetzen. Der Operateur selbst muss sich verpflichtet sehen, sich um die Details des gesamten Vorbereitungs-, Operations- und Nachsorgeprozesses zu kümmern. Schon 1888 äusserte sich Hirschberg hierzu:

«Mit gläubigem Vertrauen haben manche Fachgenossen als antiseptisch, d.h. keimtötend, solche Mittel bezeichnet, die dem reinen Wasser höchstens gleichwertig sind.»

Hirschberg betont das aseptische Vorgehen. Er weist darauf hin, dass keimtötende Mittel bei entsprechender Konzentration das Auge schädigen, und dass man sich auf nichts verlassen kann, weder auf den Fabrikanten von Verbandsstoffen noch auf den Apotheker.

Zweifellos angeregt durch ihren Lehrer, haben sich auch zwei Schüler Alfred Graefes mit eigenen Untersuchungen zur Antisepsis befasst. Es sind dies Conrad Fröhlich (1880, 1901), später Augenarzt in Berlin, und der Zürcher Ordinarius Otto Haab $(1885,1899)$.

Wie eine synoptische Beurteilung der grundlegenden Idee Alfred Graefes, der Einführung der Antisepsis in die Ophthalmologie, kann man Sätze aus einer anderen Arbeit Hirschbergs, die 1898 - ein Jahr vor Graefes Tod erschien, auffassen:

«Natürlich, nach der bakteriologischen Forschung ist die glückliche unbefangene Zeit zu Grabe getragen. Aber mit dem Gefühl grösserer Verantwortlichkeit ist auch befriedigende Sicherheit des Erfolges bei uns eingezogen. Hüten wir uns vor Einbildungen».

und weiter:

«Noch nie habe ich, und mein Auge ist scharf, das Barthaar eines Arztes auf dem Operationsgebiet entdeckt. Befolgen wir die hippokratische Regel, d.h. möglichst schweigend unsere wichtigen Handarbeiten zu verrichten, vermeiden wir namentlich dabei alle sprudelnde Beredsamkeit. So können die Mundbinden zu den Handschuhen in ein Museum getan werden, das den Urenkeln zur Erbauung dienen wird.»

Die Frage, warum gerade Alfred Graefe $(1878,1884,1887,1889,1891)-$ eigentlich der Spezialist für Motilitätsstörungen seiner Zeit - sich so intensiv mit der Problematik der Antiseptik befasst hat, muss letztlich offen bleiben. 
Sein offensichtlich gutes Verhältnis zum damaligen halleschen Ordinarius für Chirurgie, Richard v. Volkmann (1830-1889), der sich von seiten der allgemeinen Chirurgie frühzeitig mit dem Listerschen Verfahren vertraut machte, könnte ein auslösendes Moment hierfür gewesen sein. Volkmann gehörte noch zu jener Generation von Chirurgen, die zuvor die Augenheilkunde mit vertraten. Im Vorlesungsverzeichnis findet man eine diesbezügliche Ankündigung noch im Sommersemester 1858 in Halle (Eulner und Sachsenweger 1958). Beide Professoren waren Begründer des Halleschen Ärztevereins und dort führend tätig. In den Vorlesungslisten vermisst man jedoch Hinweise auf einen diesbezüglichen Gedankenaustausch. Wohl aber findet sich in der von Richard v. Volkmann herausgegebenen «Sammlung klinischer Vorträge in Verbindung mit deutschen Klinikern» ein Artikel von Alfred Graefe (1881) mit dem Thema «Über kaustische und antiseptische Behandlung der Konjunktivalentzündungen mit besonderer Berücksichtigung der Blenorrhoea neonatorum», dem ein abschliessendes, die Problematik der Antisepsis auch aus heutiger Sicht noch gut charakterisierendes Zitat zu entnehmen ist :

«Wie weit sich unsere, auf die Antiseptika gesetzten Hoffnungen erfüllen werden und die Modalität der Anwendung derselben sich als die zweckdienlichste empfehlen wird, das befindet sich z. T. im Schosse der Zukunft. Die chaotische Verwirrung, in der sich gegenwärtig nicht allein die Anpreisungen der Mittel, sondern auch die Indikationsstellungen kreuzen, müssen vor allem beklemmend auf den jüngeren Kollegen wirken, der die feste Richtschnur seines Handelns noch nicht gefunden hat und eine solche bei uns sucht.» 


\section{Literatur}

Abadie, Ch., Moyens de combattre les accidents de suppuration consécutifs à l'opération de la cataracte. Ann. d'ocul. 1883, ref. Cbl. prakt. Augenheilk. 9 (1885), 152.

Arlt, F., zit. n. Eulner, H., u. R.Sachsenweger, Die Augenheilkunde an der Universität Halle. Wiss. Z. Univ. Halle, Math.-Nat. R. 7 (1958), 397-401.

Birnbacher, A., Über die Anwendung der Sterilisation durch Hitze auf die oculistische Asepsis. Cbl. prakt. Augenheilk. 9 (1885), 225-228.

Bjerrum, J., Statistik über inflammatorische Fälle nach Cataractextractionen. Nord. ophthalmol. tidsskrift 1 (1888), 138-143, ref. Cbl. prakt. Augenheilk. 12 (1888), 381.

Bribosia, M., Du pansement antiseptique après les opérations des yeux. Bull. de l'Acad. Belg. 1879 (13/12), ref. Cbl. prakt. Augenheilk. 4 (1880) 111-112.

Eulner, H., u. R. Sachsenweger, Die Augenheilkunde an der Universität Halle. Wiss. Z. Univ. Halle, Math.-Nat. R. 7, (1958), 397-401.

Fröhlich, C., Über Antisepsis bei Augenoperationen. Klin. Mbl. Augenheilk. 18 (1880) 115-122.

Fröhlich, C., Zur offenen Wundbehandlung der Staroperation. Klin. Mbl. Augenheilk. 39/1 (1901), 257-275.

Graefe, A., Die antiseptische Wundbehandlung bei Cataract-Extraction. A. v. Graefes Arch. Ophthalmol. 24/1 (1878), 233-251.

Graefe, A., Über caustische und antiseptische Behandlung der Conjunctivalentzündung mit besonderer Berücksichtigung der Blennorrhoea neonatorum. In: Sammlung klinischer Vorträge, Hrsg. R. Volkmann, 1881.

Graefe, A., Wundbehandlung bei Augen-Operationen mit besonderer Berücksichtigung der Staar-Extraktion. - Operation unreifer Staare. A. v. Graefes Arch. Ophthalmol. 33/4 (1884), 211234.

Graefe, A., Fortgesetzter Bericht über die mittels antiseptischer Wundbehandlung erzielten Erfolge der Staaroperationen. A. v. Graefes Arch. Ophthalmol. 35/3 (1889), 248-264.

Graefe, A., Zur Wundbehandlung der Cataractextraction. Dtsch. med. Wochenschr. 17 (1891), 1193.

Haab, O., Kleinere ophthalmologische Mittheilungen. Correspondenzbl. Schweiz. Arzte, 11 (1881), 38-42, 79-81, 105-106, ref. Cbl. prakt. Augenheilk. 5 (1881), 78-79.

Haab, O., Über Ätiologie und Prophylaxe der Ophthalmo-Blennorrhoe Neugeborener. Correspondenzbl. Schweiz. Arzte 15 (1885), 7-11, 28-32.

Haab, O., Bemerkungen zur Staroperation. Cbl. prakt. Augenheilk. 15 (1891), 346-348, 365-367. Hirschberg, J., Zur Wundbehandlung des Starschnittes. Berliner klin. Wochenschr. 1888, Nr. 38, ref. Cbl. prakt. Augenheilk. 12 (1888), 313-314.

Hirschberg, J., Bemerkungen über reinliche Wundbehandlung. Dtsch. med. Wochenschr. 1898, ref. Cbl. prakt. Augenheilk. 22 (1898), 468-470.

Horner, F., Desinficierende Behandlung einiger Hornhauterkrankungen. Klin. Mbl. Augenheilk. 12 (1874), 432-438.

Kümmel, 14. Congress der deutschen Chirurgen. Cbl. prakt. Augenheilk. 9 (1885), 158.

Panas, Antisepsis in der Augenheilkunde. Cbl. prakt. Augenheilk. 9 (1885), 190.

Rosenzweig, I., Über Desinfection bei Augenoperationen. Cbl. prakt. Augenheilk. 8 (1885), 268269. 
Stefan, Ph., Weitere Erfahrungen und Studien über die Kataraktextraction, 1882-1888: Antisepsis und Technik. A. v. Graefes Arch. Ophthalmol. 35 (1889), 171-206.

Strasser, P., Zur Anwendung der Desinficientien in der Ophthalmologie. Inaug. Diss. Bern 1879, ref. Cbl. prakt. Augenheilk. 4 (1880), 112.

Waldemeyer, B., Alfred Graefe - eine biologische Betrachtung. Diplom-Arbeit, Med. Fak. Univ. Halle 1981

\section{Summary}

\section{Alfred Graefe and ophthalmological antisepsis}

The author sets forth the merits of Alfred Graefe (1830-1899) of Halle for the introduction of Lister's Antiseptic Principle in cataract surgery.

\section{Résumé}

\section{Alfred Graefe et l'antisepsie en ophtalmologie}

L'introduction du procédé listérien de l'antisepsie dans la chirurgie oculaire est due à Alfred Graefe (1830-1892). Après un bref aperçu sur sa biographie et ses travaux originaux, on passe aux discussions et aux controverses qu'il a soulevées. L'étude de son environnement scientifique comprend les bonnes relations d'Alfred Graefe avec Richard v. Volkmann (1830-1889), alors professeur ordinaire de chirurgie à Halle, dont les efforts en faveur de l'introduction de l'antisepsie dans la chirurgie générale ont été décisifs.

OMR Prof. Dr. med. habil. M. Tost

Direktor der Klinik und Poliklinik

für Augenkrankheiten Martin-Luther-Universität Halle-Wittenberg

PSF 302

D-0-4020 Halle/S. 